\title{
TITLE:
}

\section{Back pain after wild mushroom consumption.}

AUTHOR(S):

Nishiyama, K; Ohta, Y; Matsuda, N; Fujimoto, R; Koike, $\mathrm{K}$

\section{CITATION:}

Nishiyama, K ...[et al]. Back pain after wild mushroom consumption.. Emergency medicine journal : EMJ 2010, 27(1): 61-61

ISSUE DATE:

2010-01

URL:

http://hdl.handle.net/2433/97923

RIGHT: 


\section{Back pain after wild mushroom consumption}

A 62-year-old man was brought to the hospital with back pain and muscle weakness accompanied by myalgia. These symptoms developed $20 \mathrm{~h}$ after he had consumed his last meal which included the wild mushroom Russula subnigricans. The muscle weakness persisted for over a week. The maximal serum creatine kinase activity was $42689 \mathrm{U} / \mathrm{l}$. The patient did not have a history of trauma, pharmacotherapy or any other known causative factor that could explain the occurrence of rhabdomyolysis. Additional tests performed to detect the presence of parasites and other micro-organisms as well as systemic diseases were negative.

Magnetic resonance imaging (MRI) has been shown to be more sensitive than CT scanning or ultrasonography for detecting muscle abnormalities. ${ }^{1}$ MRI of the muscle using the short TI inversion recovery (STIR) technique revealed diffuse high signal intensity in the bilateral infraspinatus (white arrow) and left supraspinatus muscles (white arrowheads), confirming rhabdomyolysis.

\section{K Nishiyama, ${ }^{1}$ Y Ohta, ${ }^{1}$ N Matsuda, ${ }^{1}$ R Fujimoto, ${ }^{2}$ K Koike ${ }^{1}$ \\ ${ }^{1}$ Department of Primary Care and Emergency Medicine, Kyoto University Graduate School of Medicine, Kyoto, Japan; ${ }^{2}$ Department of Nuclear Medicine and Diagnostic Imaging, Kyoto University Graduate School of Medicine, Kyoto, Japan}

Correspondence to: Dr K Nishiyama, Department of Primary Care and Emergency Medicine, Kyoto University Graduate School of Medicine, 54, Shogoin, Kawahara-cho, Sakyo-ku, Kyoto, Japan 606-8397; keinishi@kuhp.kyoto-u.ac.jp

\section{Competing interests: None.}

Patient consent: Obtained.

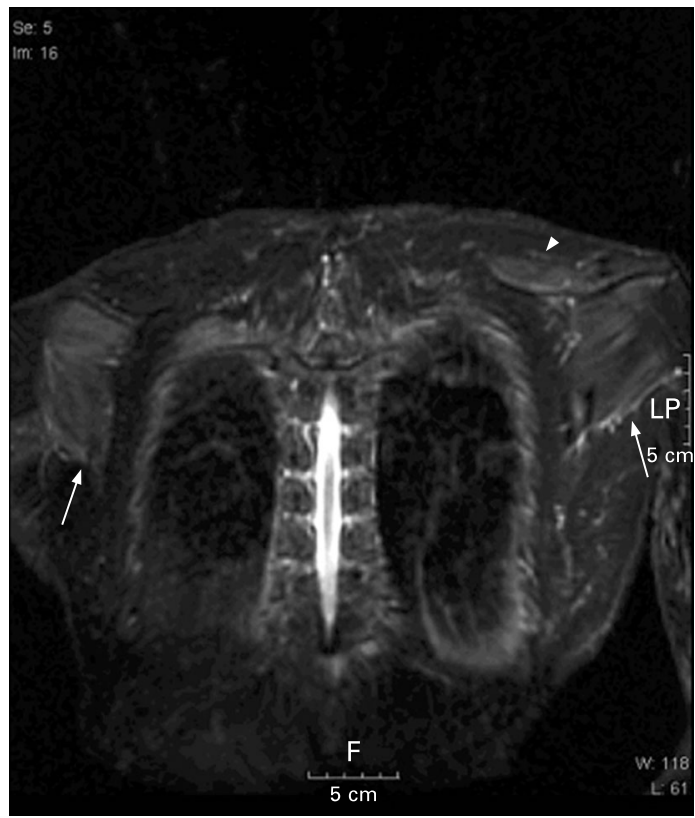

Figure 1 Muscle MRI image: short TI inversion recovery (STIR) images of bilateral shoulder showing high density areas in the bilateral infraspinatus muscle (white arrow) and left supraspinatus muscle (white arrowheads).

Accepted 2 October 2008

Emerg Med J 2009;000:000. doi:10.1136/emj.2008.066803

\section{REFERENCES}

1. Moratalla MB, Braun P, Fornas GM. Importance of MRI in the diagnosis and treatment of rhabdomyolysis. Eur $\mathrm{J}$ Radiol 2008;65:311-5. 